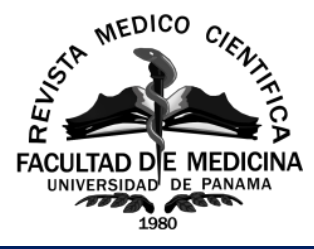

\title{
Un biólogo en la Facultad de Medicina: breve reseña biográfica de Alejandro Méndez Pereira
}

\section{A biologist in Medical School: brief biographical sketch of Alejandro Méndez Pereira}

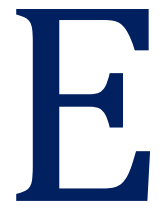

ste 2021, la Facultad de Medicina de la Universidad de Panamá cumple 70 años de fundación, motivo por el cual, se han redactado diversas alusiones al tema, entre las que podemos mencionar la del exdecano de la Facultad, Enrique Mendoza (2021) ${ }^{[1]}$, titulada "Los 70 años de fundación de la Facultad de Medicina de la Universidad de Panamá". Aquella carta al editor, publicada en esta misma revista, versa brevemente sobre la historia de la facultad, su evolución y sus desafíos en la actualidad.

Un aspecto interesante que nos motiva a escribir este documento es la somera mención de Alejandro Méndez Pereira como parte importante en la creación de la facultad, junto a sus hermanos Octavio Méndez Pereira, fundador y primer rector de la Universidad de Panamá y Gustavo Méndez Pereira, decano de la Facultad años más tarde. En esta carta no buscamos abordar la historia de la facultad, considerando que ya existe documentación de la misma; por el contrario, nuestro objetivo es rendir homenaje a su primer decano, Alejandro Méndez Pereira (ver Figura 1).

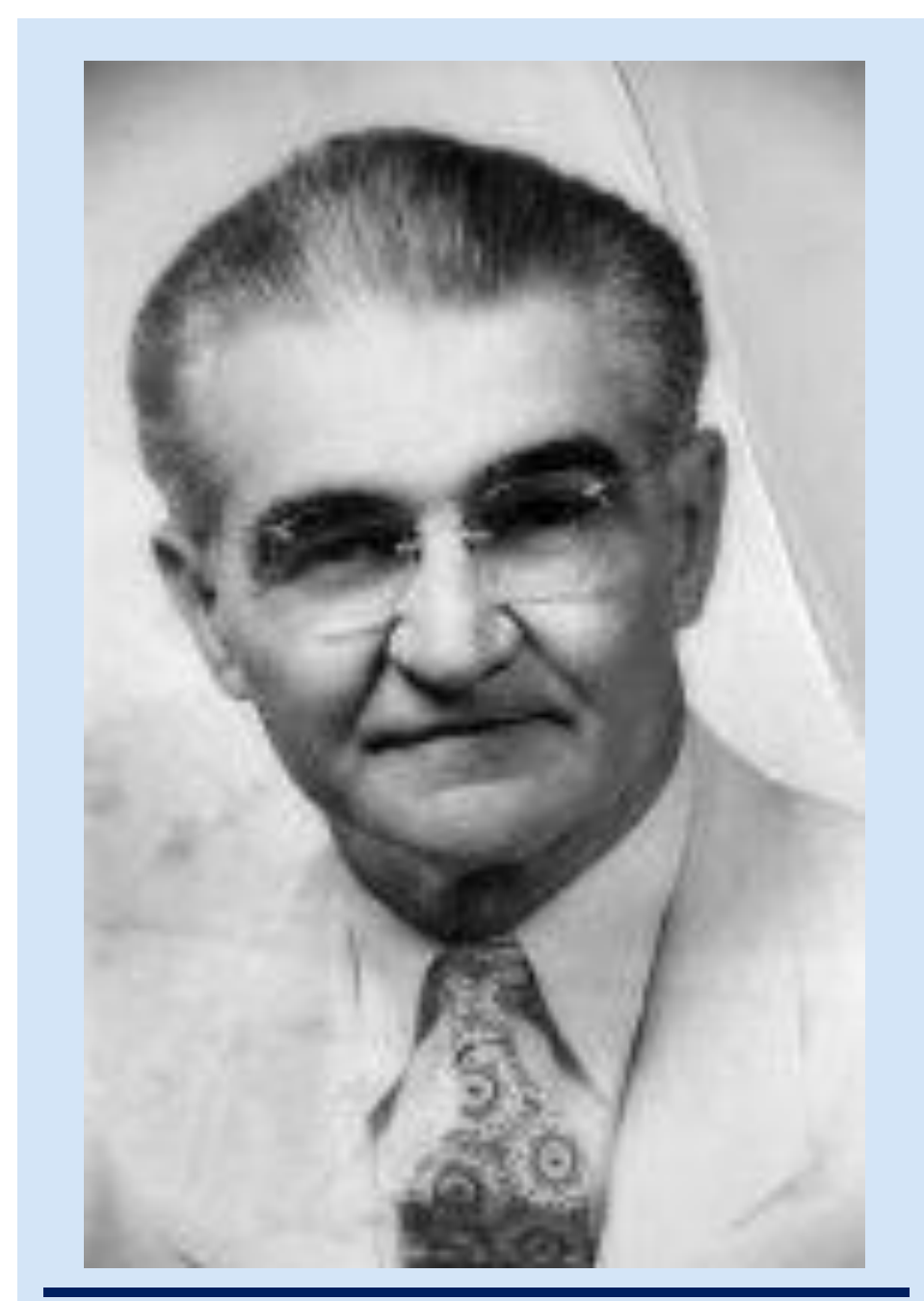

Figura 1. Profesor Alejandro Méndez Pereira

Primer decano de la Facultad de Medicina de la Universidad de Panamá.

Fuente: Alonso Roy (2001).[2] 
Alejandro Méndez Pereira se gradúa con el primer puesto de honor en el Instituto Nacional de Panamá (promoción 1915). En 1920, obtiene el título de profesor de Estado en Ciencias Biológicas en la Universidad de Chile, convirtiéndose en el primer panameño en obtener un título en Biología. En 1928, realiza estudios sobre organización de museos y laboratorios de ciencias en Estados Unidos. Recibió el grado de doctor honoris causa de la Facultad de Ciencias de la Universidad del Cuzco (Perú) en 1949 por sus investigaciones en áreas como la botánica, zoología y arqueología.[3] Entre sus labores académicas podemos mencionar que fue: profesor de Anatomía Vegetal y Botánica Farmacéutica en la Universidad de Panamá, fundador del Museo de Ciencias Naturales en 1925, siendo su primer director hasta 1936; miembro de la Asociación de Museos de San Diego (Estados Unidos), la Academia de Historia de Panamá, México, Madrid y Argentina, Sociedad Botánica de Cuba y académico correspondiente de la Academia Panameña de la Lengua.

En 1951, Alejandro Méndez Pereira funda la Escuela de Medicina de la Universidad de Panamá, que dos años más tarde se transformaría en facultad, de la cual sería su primer decano hasta junio de 1954 cuando renuncia, siendo reemplazado por el Dr. Jaime de la Guardia. Fue así como gracias a sus valiosos aportes a la enseñanza en la época, además de su ardua labor por hacer realidad lo que en ese momento parecía una utopía, se le concede el título de Decano Honorario de la Facultad de Medicina.

Finalmente, después de una próspera carrera académica, fallece el 21 de mayo de 1985. En junio de ese mismo año, la Asamblea Legislativa de Panamá crea el Premio Nacional de la Ciencia que lleva su nombre, con el propósito de honrar a panameños y panameñas que se distingan por sus ejecutorias en el campo de las ciencias y que hayan realizado aportes significativos al desarrollo nacional en las diversas áreas de la actividad científica (ver Figura 2).

\section{REFERENCIAS}

[1] Adames A, Agard E, Fernández B, Flores E, Gutiérrez J, Soler A. Ciencia, Universidad y Nación (Cien años de la República). Tecnociencia [Internet]. 2003 [citado 20 de julio de 2021];5(3):1-97. Disponible en:

https://revistas.up.ac.pa/index.php/tecnociencia/issue/vi ew/62

[2] Roy A. Historia de la Facultad: Bodas de oro de la Facultad de Medicina de la Universidad de Panamá (1951-2001). Revista Lotería. [Internet]. 2001 [citado 20 de julio de 2021];435:23-66. Disponible en: http://200.115.157.117/RevistasLoteria/435.pdf

[3] Mendoza E. Los 70 años de fundación de la Facultad de Medicina de la Universidad de Panamá. Rev. Méd. Cient. [Internet]. 2021 [citado 20 de julio de 2021];34(1):16-8. DOI: https://doi.org/10.37416/rmc.v34i1.672

[4] Ley No. 4 [Internet]. Gaceta Oficial No. 20325. 21 de junio de 1985 [citado 20 de julio de 2021]. Disponible en: https://docs.panama.justia.com/federales/leyes/4-de1985-jun-12-1985.pdf 


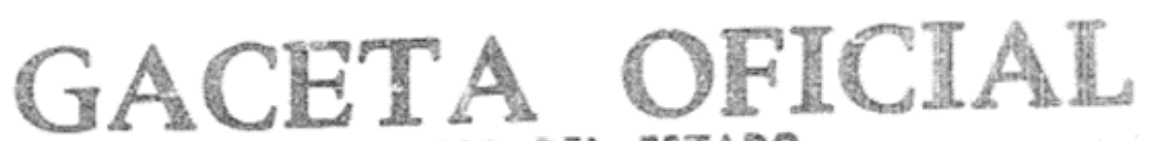 ORGANO DEI ESTADO ANC LXOXI PANAMA, R. DE P., MIERCOLES 12 DE, UNTO DE $198^{\circ}$ No 2ness \\ CONTENIDO \\ LA ASAMBLEA LEGISLATTVA \\ Ley $\mathrm{N}^{*} 4$ de 21 de mayo de 1985, por la cual se crea el Premio Nacional de Ciencia Alejand: Mendez Pereira. \\ MINISTERIO DE COMERCIO E INDUSTRIAS \\ Resoluciones $\mathrm{Y}$ 85-30 y $\mathbf{8 5 . 3 1}$ de 23 de mayo $6 \mathrm{k}$ 1535, por tas cuales se declara cue la peticionaria es elegible por la sociedad Robbins Enterprises, 5.A. \\ AVISOS Y EDICTOS \\ IA ASAMBIEA LEGISLATIVA \\ CREASE EL PREMIO NACIONAL DE CIENCIA ALEJANDRO MENUEZ PEREIRA \\ LEY 4 (de 21 de mayo de t9es) \\ Dor ia cual se erea el pragio Nacico nal do Cleneia slojastro Mandez Pe. reira. \\ LA ASAMBBLEA LECISLATVA DECRETA: \\ Annculo to crtase el Premio

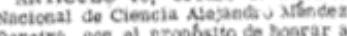

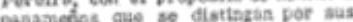 aieculertis en el cempo as as eter. ciss y qaa hayas rezHzado woortad sie: aineativos a1 desarrollo micionaton las civor as lress do ta aetifidac cieati- fiea. \\ E1 Premio Naciosal de Cleneta die- at.dre Minder. Perelira jets otcrade onualmunte, y ensistirs an entre

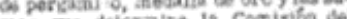 Mra qua delorwine in clatisté

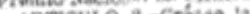 \\ ta Cosalaise cal Prezlo sacional da Ciencia Ale. gandro nes los parterses on las Unive:-

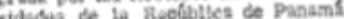 6h Rector de la vintvarzicad de pana. is is presidirs. \\ ve Mientros de esta conilaín no codr her sustinfiosos o delerar sus funci anas eot tercernge. \\ Arifecto 3.0 . Sontibsa tentra ta \\ fespunsabulited de elaterat us basen

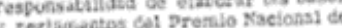 Clebeja Nejagdro Mhedea Pereira,

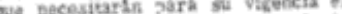 aatudio y aproba cise dal Ministerio of. conescion, ast como seleociocar atis

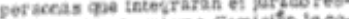 bestava A cota mistima cinsingo te eor

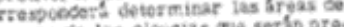 ampo oo las ctancias a d

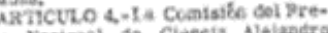 tio Nacionsi da Clegheis Nibjadr

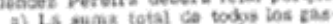 as icetosyende urcanizactin, pistici. tod $y$ administracisa del concurso, no sea mingur at diez (a0\%) por ciecto de! \\ premia za premilo sacicout de Cientia sea uno e trativisislo, satwo el caso de u. golo irabajo, compartido per bazo mis clentitioox \\ c) 21 Pramito an acjuelque todos los Hos on ta misma focha, \\ carticteo s, para lievar a cabo hit

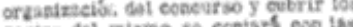

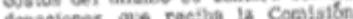 Diebas denactenes aerís cencideradas

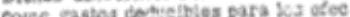

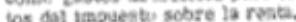

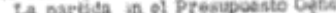

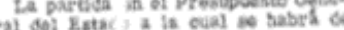 \\ imputar los gastos $y$ of greetio a che

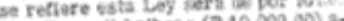

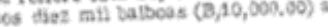 sales. \\ AgricuLo 6,-Ests Ley ocenensars a recar a pertir on su procilisiciolio.

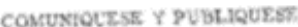 \\ Daria an la eludad ou paniats a ios 21 cisayo cen mil sovectar. toy octeats y eizco.

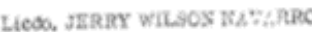 Frestonth \\ Eassao Derthen C. \\ Secrelarto Ceners!

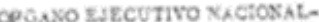

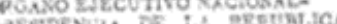

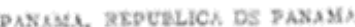 2it ve taaye de 1858 \\ NI JOLAS ARDTHO BARLETT Franomate es ia kacolica \\ MAXVEL SOT.78 D ALSCA

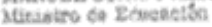

\section{Figura 2. Gaceta oficial del órgano de estado para la creación del Premio Nacional de Ciencia Alejandro Méndez Pereira}

Fuente: Ley No. 4 de 21 de junio de 1985.[4]

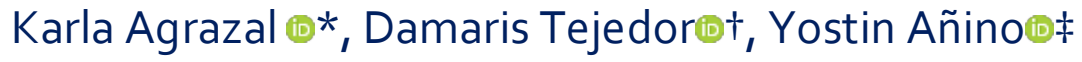

*Estudiante de Arquitectura de la Universidad de Panamá, Panamá

†Directora del Sistema de Bibliotecas de la Universidad de Panamá, Panamá

¥Profesor e Investigador del Museo de Invertebrados G.B. Fairchild de la Universidad de Panamá 